\title{
Magnetic Response of a Charged Brownian Particle Under the Action of Two AC Drive
}

\author{
Midhun A Mohan, M Sahoo* \\ Department of Physics, University of Kerala, Kariavattom, Thiruvananthapuram, India
}

Email address:

jolly.iopb@gmail.com (M Sahoo)

${ }^{*}$ Corresponding author

\section{To cite this article:}

Midhun A Mohan, M Sahoo. Magnetic Response of a Charged Brownian Particle Under the Action of Two AC Drive. International Journal of Applied Mathematics and Theoretical Physics. Vol. 7, No. 1, 2021, pp. 10-15. doi: 10.11648/j.ijamtp.20210701.12

Received: December 21, 2020; Accepted: January 6, 2021; Published: February 20, 2021

\begin{abstract}
We study the dynamics of a charged Brownian particle in a 2-D harmonic well under the action of two AC driving forces with different amplitudes as well as with a phase difference, $\phi$ between them. Interestingly we observed that the system exhibits magnetism even in the absence of magnetic field. We have exactly calculated the magnetic moment and investigated the behaviour in the presence of a linear velocity dependent force. The behaviour of the magnetic moment in various parameter regimes of the model is analyzed. The magnetic moment is found to get suppressed with increase in the amplitude of the linear velocity dependent force. Interestingly we observed that when the phase difference between the AC drives lies in between 0 and $\frac{\pi}{2}$, the system shows a paramagnetic behaviour whereas the system shows a diamagnetic behaviour when the phase difference between the AC drives lies in between $\frac{\pi}{2}$ and $\pi$. These magnetic behaviours have also been confirmed from the parametric plots. For the phase difference between 0 and $\frac{\pi}{2}$, the orbit of precission of the Brownian particle is in the clockwise direction where as for the phase difference between $\frac{\pi}{2}$ and $\pi$, the orbit of precission of the Brownian particle is in the anticlockwise direction.
\end{abstract}

Keywords: Diamagnetism, Non-equilibrium Magnetic Moment, Phase Difference, Velocity Dependent Force

\section{Introduction}

The contemporary philosophical discussions of statistical mechanics primarily focus on issues such as securing a basic foundation for thermodynamics and interpreting the probabilities in statistical physics. What philosophers mean when they say that the target of non equilibrium statistical mechanics is to account for aspects of thermodynamics. While there are a number of aspects of thermodynamics whose justification relies on non equilibrium statistical mechanics, philosophers have tended to focus on the issues to express the existence of thermodynamically irreversible processes with the underlying reversible dynamics. Equilibrium thermodynamics is based on the observation that some states of matter, which are operationally definable. The thermodynamic study of nonequilibrium systems requires more generalised concepts that deals with by the means of equilibrium thermodynamics [1-4].

The theory of Brownian motion is perhaps the simplest approximate way to treat the stochastic dynamics of nonequilibrium systems [5-7]. The most fundamental equation used to represent the dynamics of a stochastic or Brownian particle is called the Langevin equation; it mainly contain two different kinds of forces, namely frictional force or viscous force and the random force or noise. These two different kinds of forces are basically due to the intercation of the particle with the environment [8-11]. The viscous force mainly opposes the particle in a direction opposite to it's motion where as the random force try to make the movement of the particle in a random direction. Over the progress of years, there is a huge interest in the study of Brownian dynamics of a particle in various circumstances in both physics as well as in Biology. In this article, we particularly take interest in the dynamics of a charged Brownian particle under the action of two sinosoidal driving forces and in the presence of a linear velocity dependent force. Some of the recent studies are based on the stochastic dynamics of a particle in the presence of linear velocity dependent forces [12-14].

In this work, we are particularly interested in the dynamics of the particle in the nonequilibrium steady state or stationary state. We have mainly investigated the impact of the linear velocity 
dependent force on the dynamics of a charged Brownian particle under the action of two sinosoidal driving forces. Due to the circular motion of a charged Brownian particle in the presence of magnetic field, one would expect non-vanishing magnetic moment even without considering the spin of a charged particle. This delusion has been discussed by Neils Bohr and $\mathrm{H} \mathbf{J}$ van Leuween separately in there research works $[15,16]$. They had shown that in the presence of a constant magnetic field and in classical equilibrium systems, the magnetisation or the corresponding magnetic moment of a charged Brownian particle is identically zero. This is known as Bohr van Leuween $(\mathrm{BvL})$ theorem. However, the particle posses a magnetic moment when the particle is in nonequilirbium conditions [17-19]. In the present work, we show that when a charged Brownian particle is subjected to two sinosoidal driving forces with a phase difference $\phi$ between them, the system posses a nonequilibrium magnetic moment even in the absence of magnetic field and is consistent with the classical Bohr-van-Leeuwen theorem.

\section{The Model}

We consider the dynamic of a charged Brownian particle with charge $e$ in a two dimensional harmonic well in the presence of two ac drives along both $\mathrm{x}$ and $\mathrm{y}$ direction with a phase difference $\phi$ between them and an additional linear velocity dependent force $f s\left(1-\frac{v}{v_{0}}\right)$, where $v_{0}$ is the autonomous velocity [20] in both $\mathrm{x}$ and $\mathrm{y}$ direction. The motion of such a particle including inertia is described by the Langevin's equation of motion $[3,4,18,21-23]$

$$
\begin{array}{r}
m \ddot{x}=-\gamma \dot{x}-\frac{\partial U}{\partial x}+f s\left(1-\frac{\dot{x}}{v_{0}}\right)+\xi_{x}(t) \quad \text { (1) } \begin{array}{l}
\text { of this new variable, the under-dam } \\
\text { changes to, }
\end{array} \\
m z^{\prime \prime}(t)+L z^{\prime}(t)+k z(t)=A \sin (\omega t)+i B \cos (\omega t+\phi)+\mathrm{fs}^{\prime}+\xi(t)
\end{array}
$$

$$
m \ddot{y}=-\gamma \dot{y}-\frac{\partial U}{\partial y}+f s\left(1-\frac{\dot{y}}{v_{0}}\right)+\xi_{y}(t)
$$

where $m$ is the particle mass and $\xi_{\alpha}(t)$ is the random force which is a Gaussian white noise. It satisfies the properties $\langle\xi\rangle=0$ and $\left\langle\xi_{\alpha}(t) \xi_{\beta}\left(t^{\prime}\right)\right\rangle=D \delta_{\alpha \beta} \delta\left(t-t^{\prime}\right)$.

Here $\alpha, \beta=x, y$ and $\gamma$ is the frictional coefficient. The predefined factor $D=2 \gamma k_{B} T$ is the consistensy condition for a system to approach in equilibrium in the absence of a time dependent potential. The time-independent potential was considered earlier to expound the crutial role played by the boundary conditions in the celebrated theorem of Bohr van-Leeuwen in the absence of diamagnetism in classical systems[24, 25]. This interprets that the free energy of a system is independent of the presence of magnetic field. The equations of motion for the charged Brownian particle in the over-damped regime reduce to,

$$
\begin{gathered}
\gamma \dot{x}=-\frac{\partial U}{\partial x}+f s\left(1-\frac{\dot{x}}{v_{0}}\right)+\xi_{x}(t) \\
\gamma \dot{y}=-\frac{\partial U}{\partial y}+f s\left(1-\frac{\dot{y}}{v_{0}}\right)+\xi_{y}(t) \\
U(x, y, t)=\frac{1}{2} k\left(x^{2}+y^{2}\right)-A x \sin (\omega t)-B y \cos (\omega t+\phi)
\end{gathered}
$$

Here $k$ is the harmonic constant, $A$ and $B$ are the corresponding amplitudes of the ac drive forces.

\section{Results and Discussions}

In order to solve the dynamics, we have defined a new variable $z=x+i y(i=\sqrt{-1})[4,18]$ and with the help of this new variable, the under-damped equations (1) and (2)

where

$$
L=\gamma+\frac{f s}{v_{0}}, f s^{\prime}=f s+i f s, \xi(t)=\xi_{x}(t)+i \xi_{y}(t)
$$

The formal solution of above coupled equations can be obtained as,

$$
\begin{aligned}
z(t)=z_{0} e^{-\frac{(L-p) t}{m}} & +e^{-\frac{(L-p) t}{m}} \int_{0}^{t} \frac{e^{-\frac{(p-L) t^{\prime}}{2 m}}\left(i B \cos \left(\omega t^{\prime}+\phi\right)+\xi\left(t^{\prime}\right)+A \sin \left(\omega t^{\prime}\right)+\mathrm{fs}^{\prime}\right)}{p} d t^{\prime} \\
& -e^{-\frac{(L+p) t}{2 m}} \int_{0}^{t} \frac{e^{\frac{(L+p) t^{\prime}}{2 m}}\left(i B \cos \left(\omega t^{\prime}+\phi\right)+\xi\left(t^{\prime}\right)+A \sin \left(\omega t^{\prime}\right)+\mathrm{fs}^{\prime}\right)}{p} d t^{\prime}
\end{aligned}
$$

where $p=\sqrt{L^{2}-4 k m}$ where at time $t=0$ the initial condition is taken as $z_{0}=x_{0}+i y_{0}$

\subsection{Non-Equilibrium Magnetic Moment}

The averaged magnetic moment over a single period $\left(\frac{2 \pi}{\omega}\right)$ of the drive can be calculated as $[3,18,24]$

$$
<M>=\lim _{t \rightarrow \infty}\left[-\frac{e}{2 m c} \frac{\omega}{2 \pi} \int_{t}^{t+\frac{2 \pi}{\omega}}<\vec{r} \times \vec{v}>d t^{\prime}\right]
$$

Both $\vec{r}$ and $\vec{v}$ can be obtained from the solutions of the dynamics and substituting $\vec{r}$ and $\vec{v}$ in above equation, we get the nonequilibrium magnetic moment as

$$
<M>=\frac{\frac{|e|}{2 m c} \frac{A B}{m^{2}} \omega \cos (\phi)}{\left(L^{2} \omega^{2}+\left(\omega^{2}-\Omega^{2}\right)^{2}\right)}
$$




$$
L=\Gamma+\frac{f s}{m v_{0}}, \Gamma=\frac{\gamma}{m}
$$

We have plotted the dimensionless magnetic moment $(\langle M\rangle=$ $\left.\left(\frac{\langle M\rangle c \gamma^{3}}{e}\right)\right)$ as a function of dimensionless frequency $\omega$ for different values of phase difference $\phi$ and $f s$. Here we have set $A, B, \gamma, v_{0}$ and $m$ as unity.

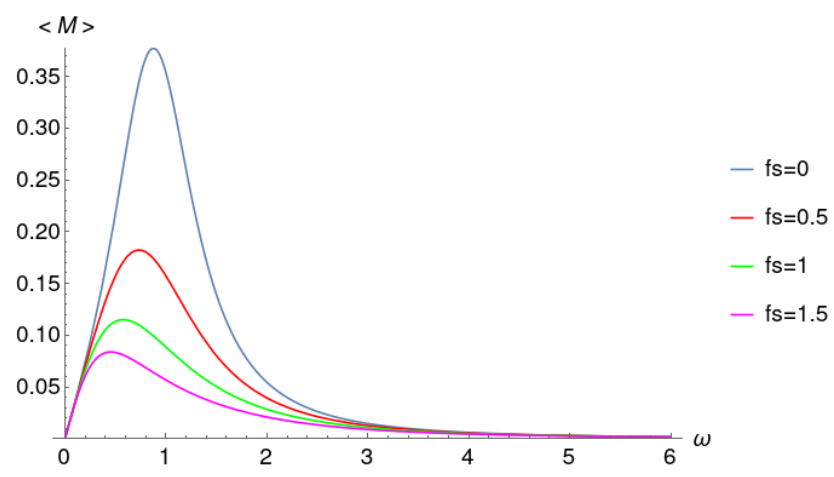

(a) $\phi=45^{\circ}$

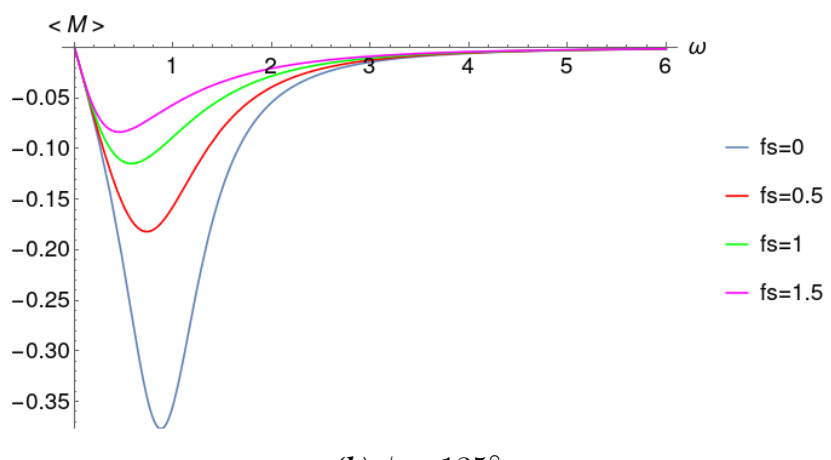

(b) $\phi=135^{\circ}$

Figure 1. The plot of dimensionless $\langle M\rangle$ versus sinusoidal frequency $\omega$ for different values of $f_{s}$ and for two different values of phase difference $\phi$.

In Figure 1(a) and Figure 1(b), we have plotted the nonequilibrium magnetic moment, $\langle M\rangle$ as a function of the frequency of the drive for two different phases. From the calculation of $\langle M\rangle$ and from the above plots, we conclude with some observations. In a non-equilibrium state, we obtain a non zero magnetic moment, and this does not violate the well known Bohr-van Leeuwen theorem as it is valid only for systems in thermodynamic equilibrium. $\langle M\rangle$ goes to zero as $\omega \rightarrow 0$, and this limit corresponds to an equilibrium state. For both small and large value of $\omega,\langle M\rangle$ vanishes that is $\langle M\rangle \rightarrow 0$ as $\omega \rightarrow 0$ and $\omega \rightarrow \infty$. For all the values of the frequency of the drive, $\omega$, the $\langle M\rangle$ is positive when the phase difference between the driving forces are 45 and hence the system shows a paramagnetic behaviour. However the $\langle M\rangle$ is negative for all the values of the frequency of the drive when $\phi$ is 135 and hence the system is diamagnetic in nature. $\langle M\rangle$ exhibits a peak as a function of $\omega$. For a fixed $f_{s}$ value, the $\langle M\rangle$ first increases, shows a peak and then decreases with $\omega$. For $f_{s}=0$, the $\langle M\rangle$ shows a peak exactly at $\omega=1$. That means at this point the systems exhbits a resonance kind of behaviour. As a result the $\langle M\rangle$ shows a maximum exactly when the freqency of the drives matches with the natural frequency of the particle, i.e., at $\omega=\sqrt{ }\left(\frac{k}{m}\right)$. The peak value of $\langle M\rangle$ gets suppressed with increase in $f s$. At the same time, the peaks get shifted towards left with increase in $f_{s}$ value. This indicates that slow varying driving force is required in order to have a maximum value in the magnetic moment with increase in the linear velocity dependent force.

The similar observations have been drawn when the phase difference is $\phi=135$. $\langle M\rangle$ exhibits a maximum as a function of $\omega$ in the negative direction. This confirms the diamagnetic behaviour of the system. The peaks of diamagnetic regime also decrease with increase in the value of $f_{s}$. The magnetic moment shows a transition with the phase difference between the two driving forces, i.e., from paramagnetic to diamagnetic behaviour at $\phi=90^{\circ}$. So the systems with $\phi<90^{\circ}$ show paramagnetic behaviour and systems with $\phi>90^{\circ}$ show diamagnetic nature.

Similarly in Figure 2, we have plotted the dimensionless magnetic moment, $\left(\langle M\rangle=\left(\frac{\langle M\rangle c \gamma^{3}}{e}\right)\right)$ as a function of frequency of the drive, $\omega$ for different values of the harmonic constant $k$ and for two different values of phase difference $\phi$. Here also we have set $A, B$ and $m$ as unity.

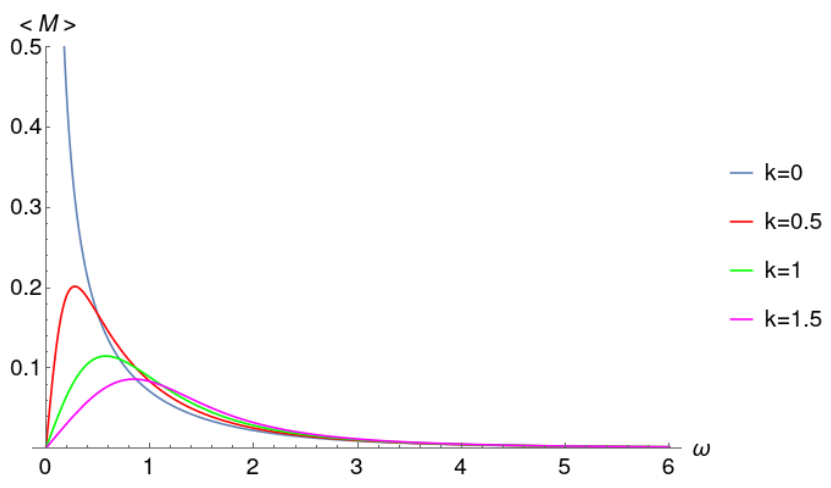

(a) $\phi=45^{\circ}$

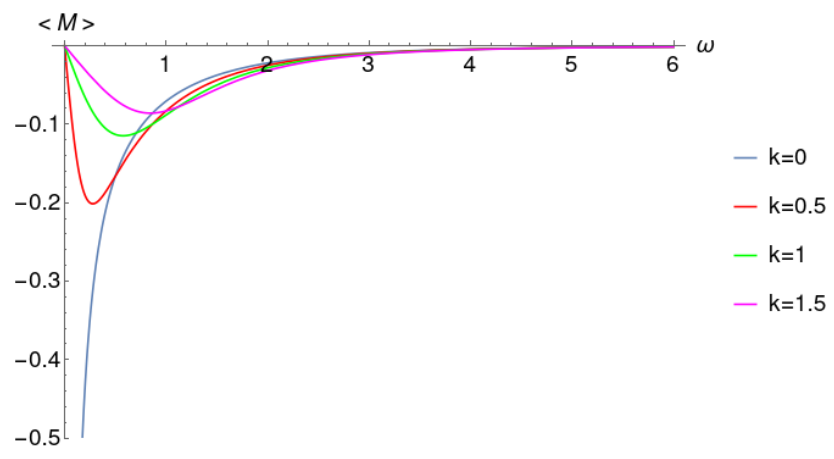

(b) $\phi=135^{\circ}$

Figure 2. The plot of average Magnetic moment $\langle M\rangle$ versus sinusoidal frequency $\omega$ for different values of $k$ and for two different phase difference $\phi$. 
From the calculation of $\langle M\rangle$ as well as from the above plot (Figure 2(a)), we observed that there exists a non zero magnetic moment in nonequilibrium state, i.e., $\langle M\rangle$ is having finite value for any finite values of the frequency of the drive other than 0 and $\infty$. In this case, the phase difference between the two driving forces is kept as 45 . In the limit of $\omega \rightarrow 0$ and $\omega \rightarrow \infty$, the $\langle M\rangle$ approaches zero value. $k \rightarrow 0$ limit basically corresponds to the free particle behaviour. In this limit, the $\langle M\rangle$ decreases with increase in the frequency of the drive and approaches zero in the large frequency limit. For a finite value of $k$, the $\langle M\rangle$ increases with increase in $\omega$, shows a maximum value and then decreases with $\omega$. The peak or maximum value in $\langle M\rangle$ shifts toward right with increase in the value of the harmonic constant $k$. This implies that with increase in $k$ value, higher value of frequency is required in order to achieve the resonant kind of behaviour, as a result the output response will be enhanced and the $\langle M\rangle$ will show a peak. Further with increase in $k$ value, the peaks get broader. This implies that the fluctuations across the minimum of the well increase with increase in the $k$ value. Similar kind of observations have been drawn when the phase difference between the driving forces is 135 . However, the $\langle M\rangle$ shows negative values for all values of frequency of the drive. This implies the system exhibits a diamagnetic nature, when the two driving forces are separated by a phase difference $\phi=135^{\circ}$.

\subsection{Parametric Plots}

In order to understand the movement or the trajectory of the particle, we have plotted the parametric plots $(y(t)$ versus $x(t)$ ) in various parameter regimes of the model. The natural frequency of the particle is nothing $\Omega=\sqrt{\frac{k}{m}}$. Due to the complex interaction of the particle in the presence of The other parameters $k, m, v_{0}$ and $\gamma$ are fixed to be as unity.

When the amplitude of both the driving forces are same and if there is no phase difference between them (i.e., if $\phi=0$ and $A=B$ ), we observed that on average the particle moves in the plane in circular orbits as expected. This implies that the particle exhibits angular momentum and hence the magnetic moment. This magnetic moment is due to the orbital motion of the particle and hence paramagnetic in nature [26]. Under the application of the linear velocity dependent force, the motion of the particle is draged in the direction of the force. As a result we observed that for the phase difference $\phi=0^{\circ}$, there is a shift of the circular orbits in the plane in the diagonal direction with increase in the value of $f_{s}$. This clearly indicates that under the action of the linear velocity dependent force, the motion of the particle on average is dragged in the direction of the force in the diagonal direction of the plane. This is because the effect of the application of the force on the motion of the particle is same in both $x$ and $y$ direction as the amplitude of the driving force are same in both $x$ and $y$ direction.

When the amplitude of driving force in the y-direction is larger than that of the amplitude of driving force in the $\mathrm{x}$ direction, i.e., for $A>B$, there is an asymmetry in the movement of the particle in the xy-plane on average. For
$A>B$, the influence of the motion of the particle is more in the direction of the amplitude of driving $A$ ( $y$-direction)as compared to the motion of the particle in the direction of amplitude of driving $B$ ( $x$-direction). As a result even if there is no phase difference between the driving forces, i.e., for $\phi=0^{\circ}$,the particle on average moves in elliptical orbits as there is an asymmetry in the motion of the particles in both the directions in the XY-plane. This corresponds to a state of non zero orbital magnetic moment. The parametric plots for different values of $f_{s}$ confirms that there is a shift of the plots in the plane with increase in $f_{s}$ value. In addition to the motion of particle in the xy plane, it follows the direction of linear velocity dependent force, that's why there is a shift in the parametric plots with increase in $f_{s}$ value.

Similarly when the amplitude of driving force in the $\mathrm{x}$ direction is smaller than that of the amplitude of driving in the y-direction, i.e., for $A<B$, the motion of the particle is more favoured in the $\mathrm{x}$-direction as compared to the $\mathrm{y}$-direction. As a result there is an asymmetry in the motion of the particle in the $\mathrm{XY}$ plane. In the presence the sinosoidal driving forces in both the directions, the particle exhibits orbital motion and hence posseses the orbital angular momentum. However the orbit of motion is elliptic in nature due to the presence of assymetry in the motion. In the presence of linear velocity dependent force, the particle follows the direction of velocity dependent force in addition to it's motion in the xy-plane. These behaviour can be confirmed from the parametric plots (Figure 3(c)). With increase in the value of $f_{s}$, there is shift of the orbits in the diagonal direction of the xy-plane.

From the parametric plots (Figure 4(a) and Figure 4(b)), it is observed that when the phase difference between the driving forces is $\phi=45^{\circ}$, the orbit of motion is elliptic in nature even if the amplitude of drivings in both $x$ and $y$ are same. Most importantly from the parameteric plots we can notice that the particle precesses in clockwise direction in an elliptic orbit. This implies that particle exhbits a positive magnetic moment and hence the system is paramagnetic in nature. This is a clear confirmation of the results obtained in Figure 1(a) and Figure 2(a) respectively. Moreover, with increase in $f_{s}$ value, there is a shift of the orbits in xy-plane. This indicates that in addition to the motion of the particles in an elliptic orbit, the particle takes the advantage of the linear velocity dependent force and follows the direction of $f_{s}$ resulting a shift of orbits in the xy-plane with increase in $f_{s}$. Similarly the parametric plots from Figure 4(b), it is reflected that when the amplitude of two driving forces are same and if they are separated by a phase difference $\phi=135^{\circ}$, the particle precesses in anticlockwise direction in an elliptic orbit and hence posses a negative magnetic moment. As a result the system shows diamagnetic behaviour. This is clear confirmation of the results already obtained from Figure 1(b) and Figure 2(b). 


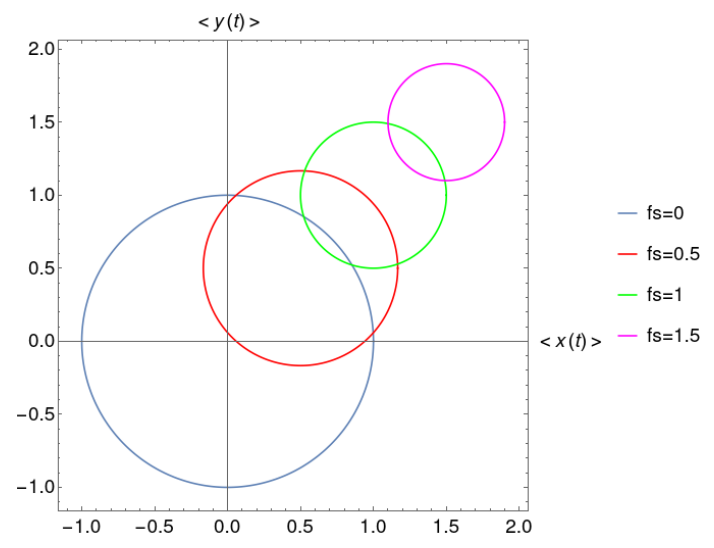

(a) $A=B, \phi=0^{\circ}$

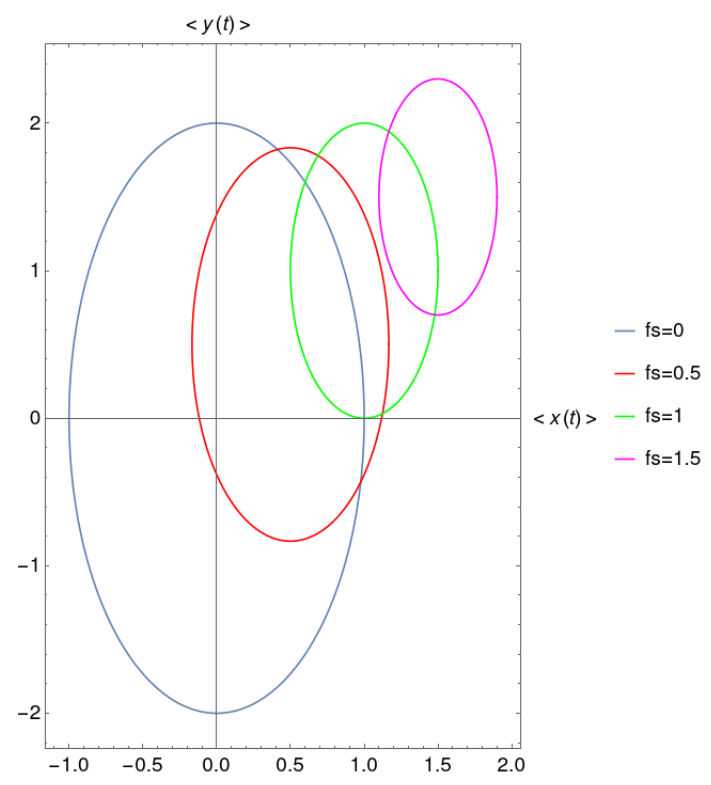

(b) $A>B, \phi=0^{\circ}$

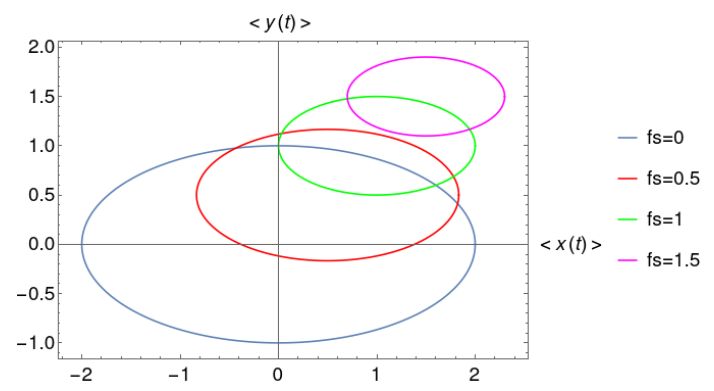

(c) $A<B, \phi=0^{\circ}$

Figure 3. Parametric plots $(\langle y(t)\rangle v s\langle x(t)\rangle)$ for different values of $f_{s}$ and for different situations for the amplitudes of drivings in $(a),(b)$ and $(c)$ respectively when the two external driving forces are separated by a phase difference $\phi=0^{\circ}$. The other parameters $k, \gamma$ and $m$ are fixed to unity.

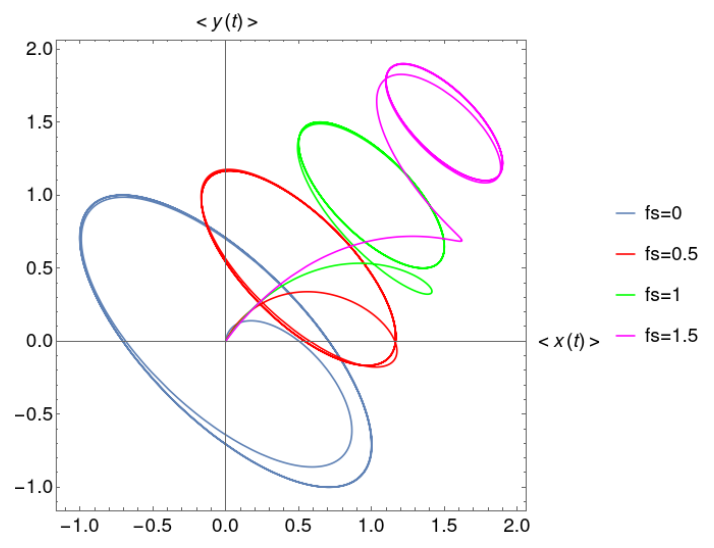

(a) $\phi=45^{\circ}$

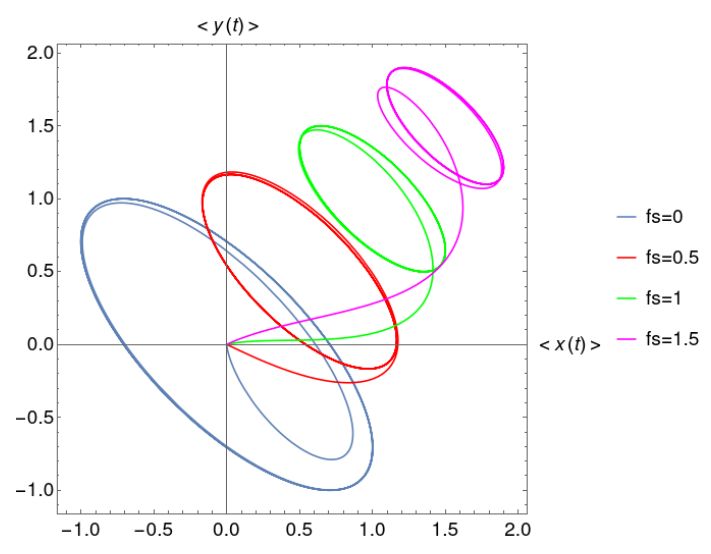

(b) $\phi=135^{\circ}$

Figure 4. Parametric plots $\langle y(t)\rangle$ versus $\langle x(t)\rangle$ for different values of $f_{s}$ and for phase difference $\phi=45^{\circ}$ in Figure 4(a) and for the phase difference $\phi=135^{\circ}$ in Figure 4(b). The amplitude of drivings remain same in both the cases. The other parameters $k, \gamma$ and $m$ are fixed to unity.

\section{Conclusion}

In conclusion, we have studied the dynamics of a charged Brownian particle in the presence of two sinosoidal driving forces of different amplitudes but with a phase difference, $\phi$ when the particle is confined in a $2 \mathrm{D}$ harmonic well and further subjected to a linear velocity dependent force. Interestingly here we observe that the particle posses an angular momentum in the presence of two sinosoidal driving forces even in the absence of magnetic field, hence shows magnetic behaviour. Further, there is a transition of the magnetic behaviour of the system at $\phi=90^{\circ}$. For $\phi<90^{\circ}$, the system is paramagnetic in nature and for $\phi>90^{\circ}$, the system is diamagnetic in nature. For both the phases, we have exactly investigated the parametric plots and analyzed the orbiting behavior of the particles. It is confirmed that for the phase difference $\phi<90^{\circ}$, the particle precesses in an elliptic orbit in the clockwise direction, reflecting the paramagnetic nature of the system. For the phase difference $\phi>90^{\circ}$, the particle precesses in an elliptic orbit with anticlockwise direction, reflecting the 
diamagnetic nature of the system. Moreover,in the presence of linear velocity dependent force the particle follows the direction of force as a result we observe a shift in the orbits in the xyplane with $f_{s}$.

\section{Acknowledgements}

MS thanks the UGC Faculty recharge program for the financial support.

\section{References}

[1] P. Maragakis, F. Ritort, C. Bustamante, M. Karplus, and G. E. Crooks, "Bayesian estimates of free energies from nonequilibrium work data in the presence of instrument noise," The Journal of chemical physics, vol. 129, no. 2, p. 07B609, 2008.

[2] K. Mallick, "Some recent developments in nonequilibrium statistical physics," Pramana,vol. 73, no. 3, p. $417,2009$.

[3] A. Jayannavar and N. Kumar, ”Orbital diamagnetism of a charged brownian particle undergoing a birth-death process,' Journal of Physics A: Mathematical and General, vol. 14, no. 6,p. 1399, 1981.

[4] A. Jayannavar and M. Sahoo, "Charged particle in a magnetic field: Jarzynski equality,'Physical Review E, vol. 75, no. 3, p. 032102, 2007.

[5] R. Kubo, M. Toda, and N. Hashitsume,"Statistical physics II: nonequilibrium statistical mechanics", vol. 31, Springer Science '\&' Business Media, 2012.

[6] G. T. Moore and M. O. Scully, "Frontiers of nonequilibrium statistical physics", vol. 135, Springer Science '\&' Business Media, 2012.

[7] L. Stella, C. Lorenz, and L. Kantorovich, "Generalized langevin equation: An efficient approach to nonequilibrium molecular dynamics of open systems," Physical Review B, vol. 89,no. 13, p. 134303, 2014.

[8] R. Zwanzig, "Nonlinear generalized langevin equations," Journal of Statistical Physics, vol. 9, no. 3, pp. 215-220, 1973.

[9] B. G. de Grooth, "A simple model for brownian motion leading to the langevin equation," American journal of physics, vol. 67, no. 12, pp. 1248-1252, 1999.

[10] D. Rings, R. Schachoff, M. Selmke, F. Cichos, and K. Kroy, "Hot brownian motion," Physical review letters, vol. 105, no. 9, p. 090604, 2010.

[11] N. Pottier, Nonequilibrium statistical physics: linear irreversible processes. Oxford University Press, 2009.

[12] L. Chen, "Nonequilibrium fluctuation-dissipation theorem of brownian dynamics," The Journal of Chemical Physics, vol. 129, no. 14, p. 144113, 2008.
[13] J. Liphardt, S. Dumont, S. B. Smith, I. Tinoco, and C. Bustamante, "Equilibrium information from nonequilibrium measurements in an experimental test of jarzynski equality,” Science, vol. 296, no. 5574, pp. 1832 1835, 2002.

[14] T. Sagawa and M. Ueda, "Generalized jarzynski equality under nonequilibrium feedback control," Physical review letters, vol. 104, no. 9, p. 090602, 2010.

[15] J. R. Roth, Plasma stability and the Bohr-van Leeuwen theorem, vol. 3880. Citeseer, 1967.

[16] H. Essen and M. C. Fiolhais, "Meissner effect, diamagnetism, and classical physics review," American Journal of Physics, vol. 80, no. 2, pp. 164-169, 2012.

[17] S. Chandrasekhar, "Stochastic problems in physics and astronomy," Reviews of modern physics, vol. 15, no. 1, p. $1,1943$.

[18] A. Jayannavar and M. Sahoo, "Fluctuation theorems and orbital magnetism in nonequilibrium state," Pramana, vol. 70, no. 2, pp. 201-210, 2008.

[19] O. Chubykalo, R. Smirnov-Rueda, J. Gonzalez, M. Wongsam, R. W. Chantrell, and U. Nowak, "Brownian dynamics approach to interacting magnetic moments,' Journal of magnetism and magnetic materials, vol. 266, no. 1-2, pp. 28-35, 2003.

[20] C. Ganguly and D. Chaudhuri, "Stochastic thermodynamics of active brownian particles,'Physical Review E, vol. 88, no. 3, p. 032102, 2013.

[21] Zhao, Zhiyuan, et al. "Brownian dynamics simulations of magnetic nanoparticles captured in strong magnetic field gradients." The Journal of Physical Chemistry C 121.1 : 801-810, 2017.

[22] Kahmann, Tamara, and Frank Ludwig. "Magnetic field dependence of the effective magnetic moment of multicore nanoparticles." Journal of Applied Physics 127.23, 2020 .

[23] K. Sekimoto, "Langevin equation and thermodynamics," Progress of Theoretical Physics Supplement, vol. 130, pp. 17-27, 1998.

[24] T. Kaplan and S. Mahanti, "On the bohr-van leeuwen theorem, the non-existence of classical magnetism in thermal equilibrium," EPL (Europhysics Letters), vol. 87, no. 1, p. 17002, 2009.

[25] R. E. Peierls and R. Peierls, Surprises in theoretical physics. Princeton University Press, 1979.

[26] A. A. Deshpande and N. Kumar, "Classical orbital paramagnetism in non-equilibrium steady state," Journal of Astrophysics and Astronomy, vol. 38, no. 3, p. 57, 2017. 